\title{
Study of Muon Monitor Data to Maintain the Quality of the NuMI Neutrino Beam at Fermilab
}

Don Athula Wickremasinghe and Katsuya Yonehara

\section{Introduction}

A muon monitor which measures muon beam profile, is a key beam element to maintain the quality of muon neutrino beam. Three arrays of muon monitors located in the downstream of the hadro absorber provide the measurements of the primary beam quality. We studied the response of muon monitors with the proton beam profile changes and focusing horn current variations. The responses of muon monitors have been used to implement Machine Learning (ML) algorithms to monitor the beam quality.

\section{NuMI neutrino beam at Fermilab}

$120 \mathrm{GeV} / \mathrm{c}$ momentum protons from the Main Injector are striking with a graphite target to produce mesons. Charged mesons are focused into the decay pipe. The decay of pions and kaons produces muons and muon-neutrinos. This muon-neutrino beam is delivered to neutrino experiments such as NOvA.

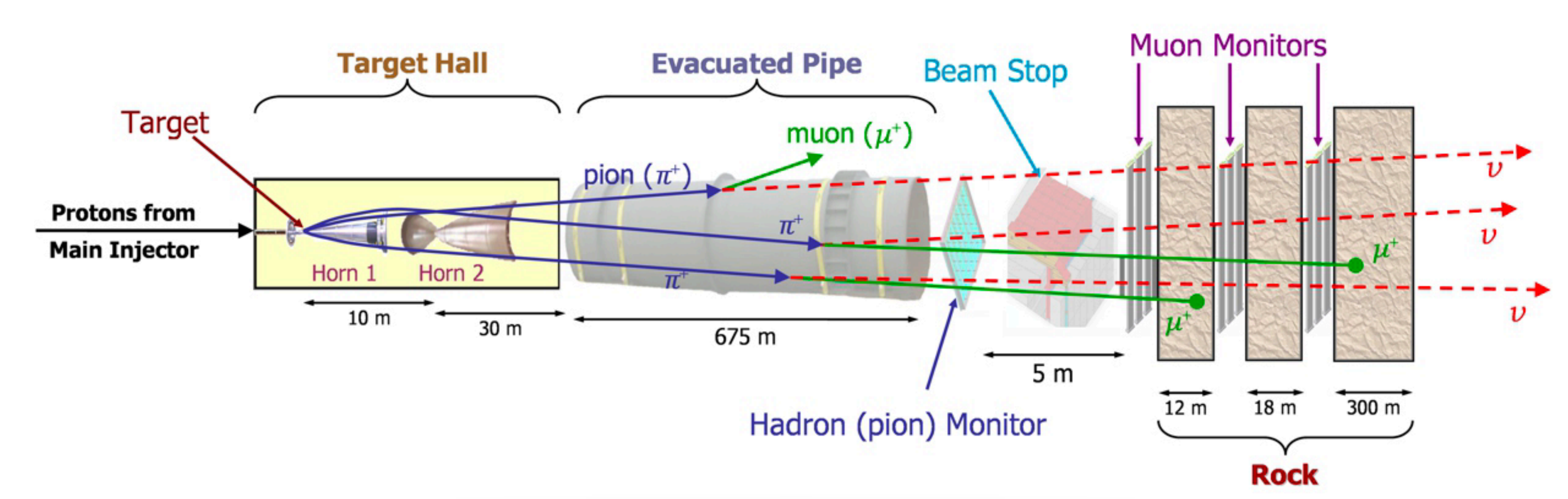

Muon Monitors

Three muon monitors are located in the downstream of the hadron absorber

- Each muon monitor consists of $9 \times 3$ arrays of ionization chambers

- Each ionization chamber consists of two parallel plate electrodes with the separation of $3 \mathbf{~ m m}$ gap

- The chambers are filled with He gas

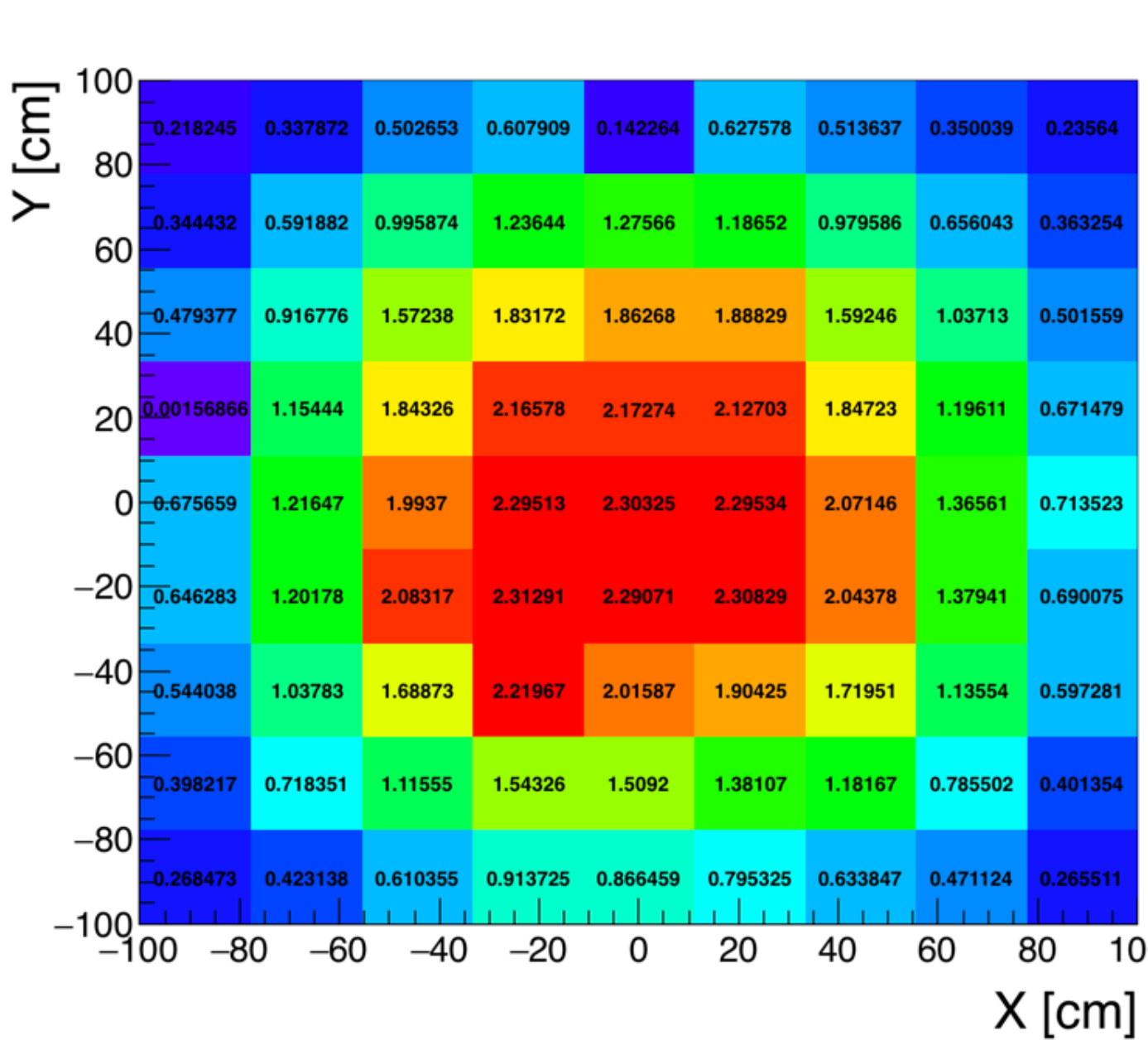

81 pixels of signal readouts on Muon Monitor 1

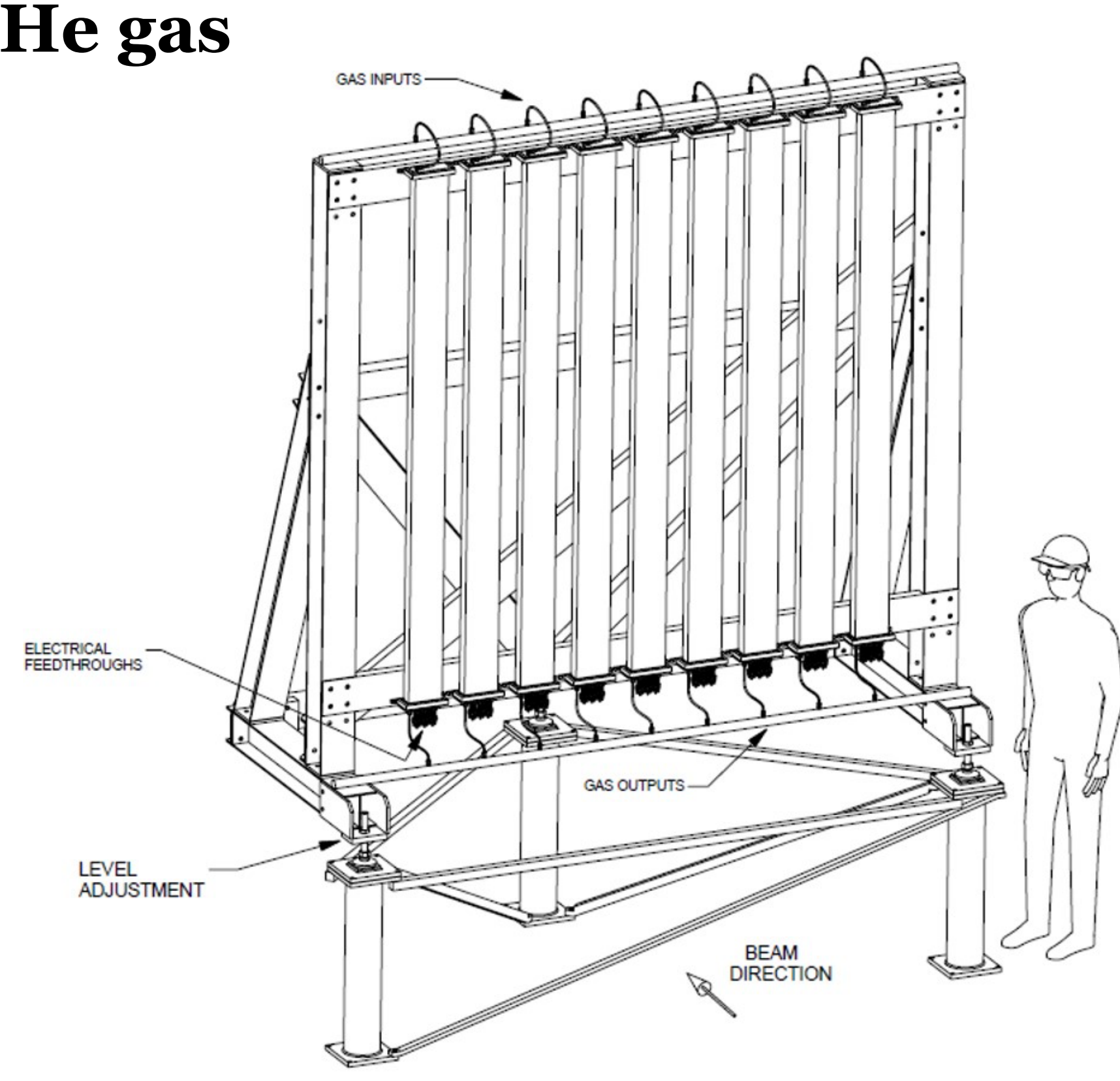

\section{Beam and Horn Current Scan Studies}

During this scan, the proton beam position has been varied horizontally and vertically to study the muon flux centroid correlation to the beam position for different horn current settings. Two BPM measurements are used to extrapolated the beam on to the target

Not to scale)

Locations of the beam position monitors 121 , TGT and the target relative to the MCzero point

Scan data: 2019-12-12

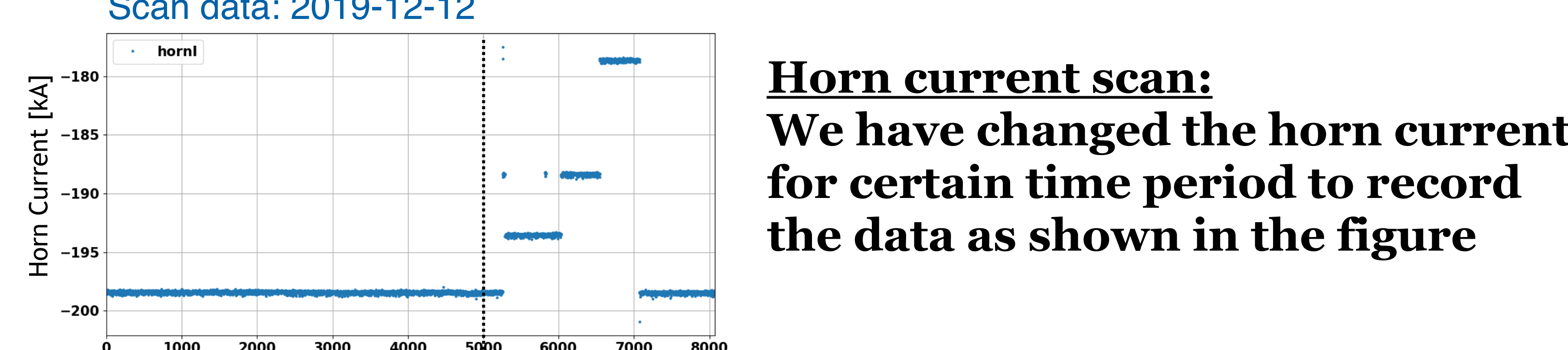

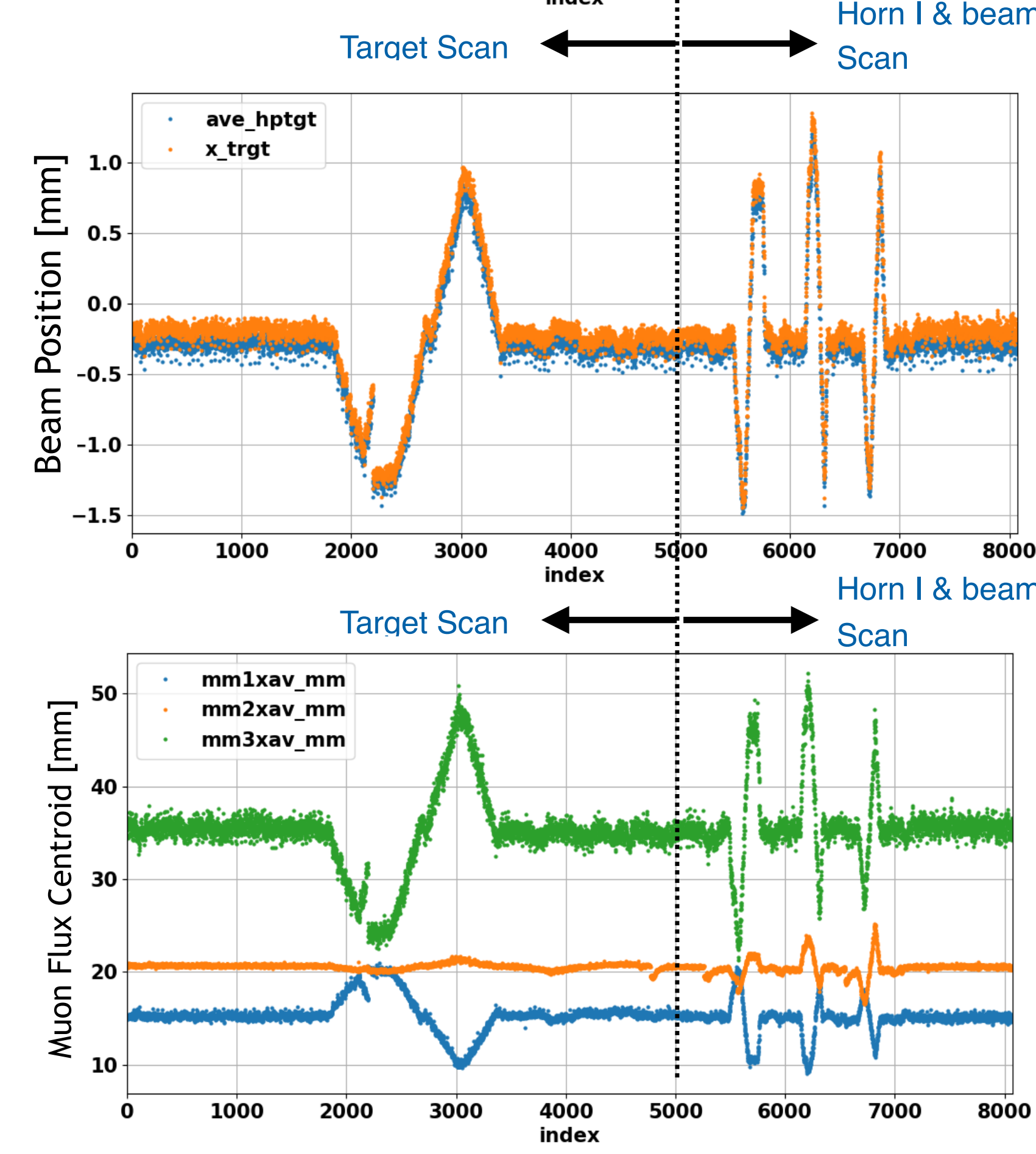

We have studied horizontal and vertical be scans with selected horn current settings

- The beam scan study shows how each muon monitor responds to the beam position varations in horizontal and vertic directions

The horn current scan shows how the horn focusing effects on the muon flux and the

This observations indicate the physics of muon flux related to the hadron produc

horn focussing and the target geometry

The study is important for neutrino experiments to model the correlation of the muon monitor observations to the neutrino
beam flux
Machine Learning Applications

Building Machine Learning tools to predict the proton beam profile and horn current changes by using muon monitor signals are helpin
us not only to understand beamline stability but also to reduce the neutrino flux systematics.

Predicting Beam Parameters and Horn Current MOTIVATION: A tool to predict beam parameters and horn current by taking account muon monitor signals

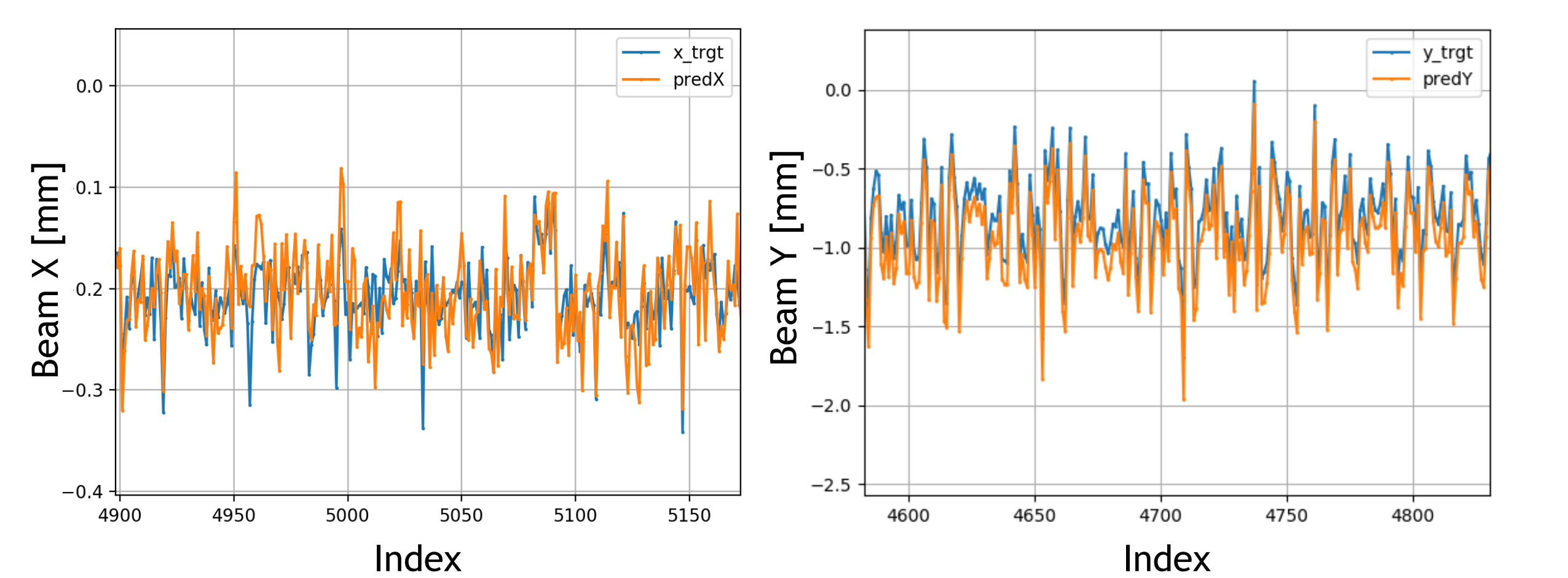

We have modeled a Neural Network to predict beam parameters

- Inputs are $81 \times 3$ pixels from all three muon

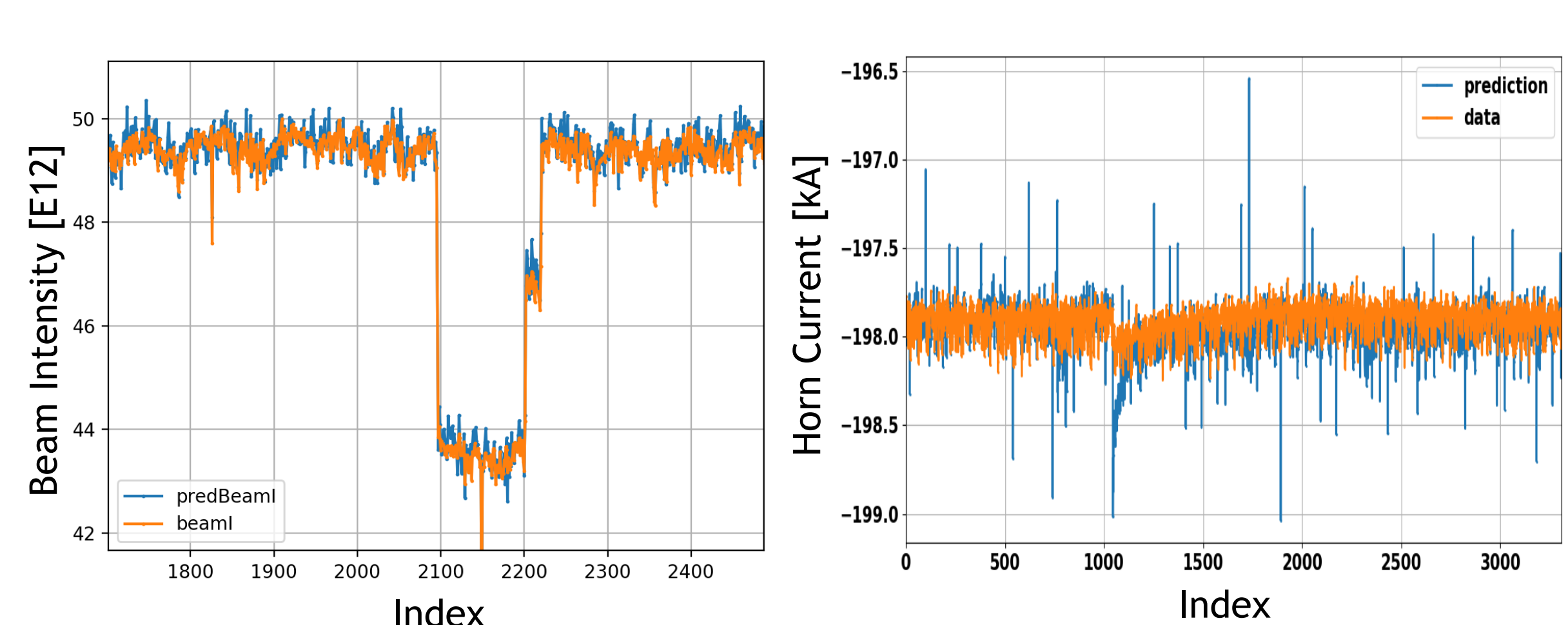
monitors

Proton beam scan:

Proton beam has been changed horizontally and vertically for selected horn current settings Muon Monitor Responses: Muon monitors are responding Mencent and eam variations

\section{Identifying / predicting Incidents}

MOTIVATION: A tool to predict and identify incidents or anomalies from the spill-by-spill. This helps operators and experts to aware about the incidents

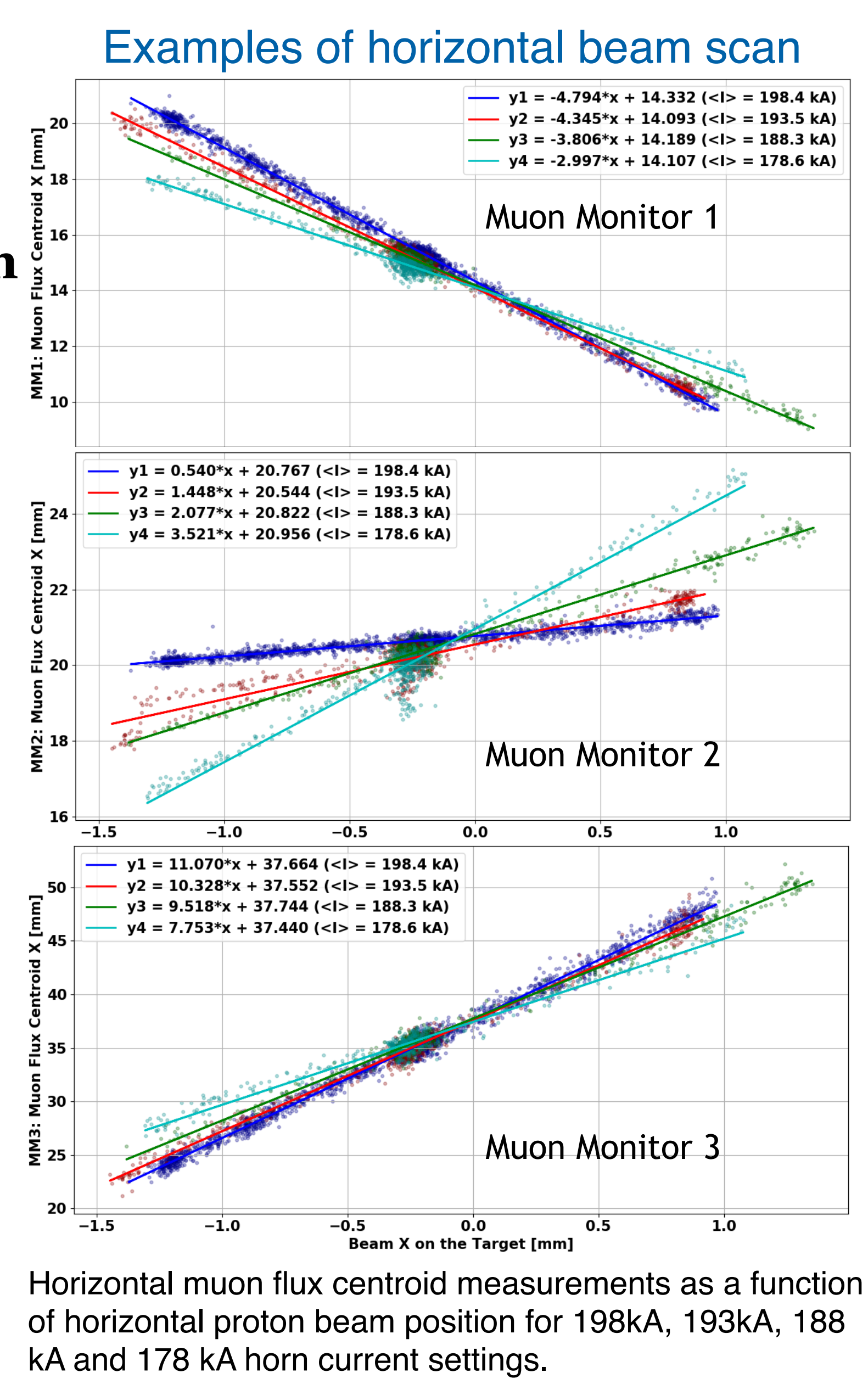

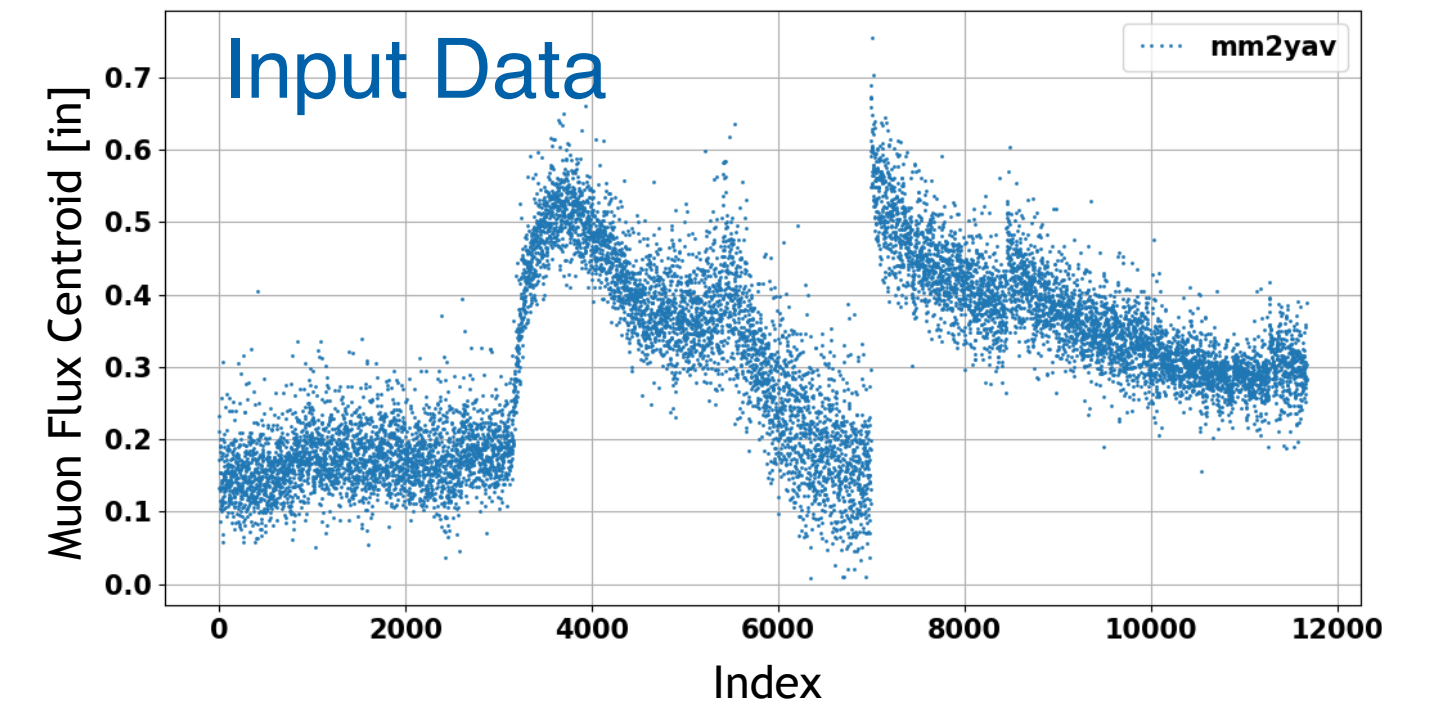

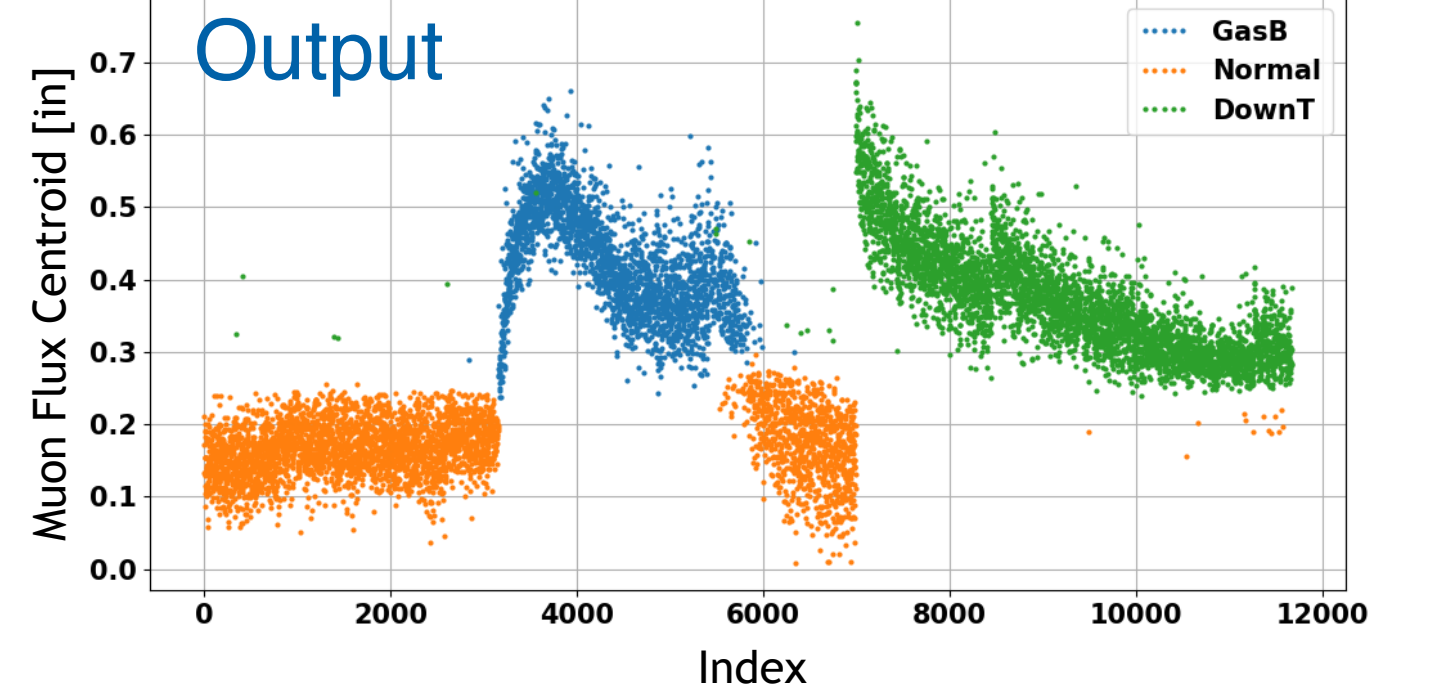

In this example, we have taken account two recognized incidents: Gas bottle incidents (GasB) and after the beam downtime behaviors (DownT) After the training, the model has predicted/identified incidents as expected

\section{Conclusion}

- Beam and horn current scan studies are helping us the understand the physics of muon flux related to the hadron production, horn focussing and the target geometry

- Tested ML applications show a good prediction accuracy

- Model tuning and building applications are ongoing projects 